\title{
ON THE STRUCTURE OF LINEAR RECURRENT ERROR-CONTROL CODES
}

\author{
MiCHEL FLIESS ${ }^{1,2}$
}

\begin{abstract}
We are extending to linear recurrent codes, i.e., to time-varying convolutional codes, most of the classic structural properties of fixed convolutional codes. We are also proposing a new connection between fixed convolutional codes and linear block codes. These results are obtained thanks to a module-theoretic framework which has been previously developed for linear control.
\end{abstract}

Mathematics Subject Classification. 93C65, 94B05, 94B10, 12H05, 13A02, $13 \mathrm{C} 05$.

Received December 2, 2001.

\section{À la mémoire du professeur Jacques-Louis LIONS}

\section{INTRODUCTION}

This paper is devoted to various aspects of convolutional codes which are with linear block codes the most popular class of error-control codes (see, e.g. $[2,4,30,31]$ ). It is based on the well known ties between convolutional codes and linear automatic control (see, e.g. [21-23,25-27,33,34,38]).

Our aim is twofold:

- we are extending to linear recurrent codes, i.e., to time-varying convolutional codes, most of the classic structural properties of fixed, i.e., time-invariant, convolutional codes (see, e.g. $[6,26,34,37]$ ). Although Shannon's channel coding theorem has been extended to time-varying convolutional codes (see [42]) and not to fixed ones, those time-varying codes were much less utilized in practice than the time-invariant ones (see, nevertheless [26]);

- the connection between fixed convolutional codes and special types of linear block codes, like cyclic codes, which has been the subject of many investigations (see, e.g. $[31,37,40]$ and the references therein), is here approached from a new perspective. Our main theorem states that for an arbitrary block code there exists a convolutional code such that all its frames (see Sect. 4.2 for a precise definition) are isomorphic to this block code. This leads to families of convolutional codes and to a feedback decoding procedure, which seem to be novel.

The relationship between those two rather independent subjects is a module-theoretic approach to linear control $[9,11,12,15,18-20]^{3}$, which has been quite useful in practice $[16-19,36]^{4}$. We are utilising some elementary

Keywords and phrases: Convolutional codes, linear recurrent codes, block codes, transducers, encoders, feedback decoding, linear systems, controllability, observability, input-output inversion, modules.

${ }^{1}$ Centre de Mathématiques et Leurs Applications, École Normale Supérieure de Cachan, 61 avenue du Président Wilson,

94235 Cachan, France; e-mail: fliess@cmla.ens-cachan.fr

2 Laboratoire GAGE, École polytechnique, 91128 Palaiseau, France.

${ }^{3}$ One should also cite some recent works of different natures on the connection between coding and control (see, e.g. $[7,24,32,38-40])$.

${ }^{4}$ See also [13] and the references therein. 
notions of difference algebra [5], homological algebra [41], and non-commutative algebra [29,35], which is most natural in the time-varying case (see, e.g. $[9,11,12,15])$.

In the first part we define, following [26], transducers, i.e., input-output systems, and study their main properties: state-variable representation, controllability, observability, transfer matrices, input-output inversion. In particular, an encoder is a right invertible transducer. The second part is devoted to codes. A code, here, is an equivalence class between encoders having the same output. We derive syndrome formers, dual codes, parity check matrices, polynomial and basic encoders, and Forney's theory in a manner which is often very short thanks to our algebraic setting. We end with the connection with block codes.

The following topics will be developed in future publications:

- constructions of cyclic-like convolutional codes, i.e., convolutional codes which thanks to the results of Section 4 will also benefit from the properties of some types of cyclic codes;

- turbo-codes [1]. They are often given by two convolutional encoders in parallel with an interleaver, and are known to be related to time-varying convolutional codes;

- non-linear tree codes, which correspond to non-linear encoders, i.e., to right invertible non-linear inputoutput systems [8] (see Sect. 2.6.2);

- cryptography is already known to be related to error-control codes (see, e.g. [45]). Encrypters will be associated to invertible square input-output systems (see Sect. 2.6.2).

\section{LiNEAR RECURRENT TRANSDUCERS}

\subsection{Algebraic preliminaries}

\subsubsection{Difference fields}

A difference field [5] is a commutative field $F$, equipped with a transformation $\delta: F \rightarrow F$, i.e., a monomorphism. Here $\delta$ should be understood as the delay operator of one unit of time. A constant is an element $c \in F$, such that $c \delta=c$ (mappings are written on the right). The subfield of constants of $F$ is the subfield of all constant elements of $F$. A field of constants is a difference field which coincide with its subfield of constants. The inversive closure $F^{\text {[5] }}$ [5, which is unique up to isomorphism, is the smallest difference overfield of $F$ such that $\delta$ is an automorphism. The difference field $F$ is said to be inversive if, and only if, $F=F^{*}$.

Example 2.1. Let $\mathbb{F}(t)$ be the field of rational functions in the indeterminate $t$ over the field $\mathbb{F}$, a finite field for instance. With the $\mathbb{F}$-automorphism $\delta: \mathbb{F}(t) \rightarrow \mathbb{F}(t), t \mapsto t-1, \mathbb{F}(t)$ becomes an inversive difference field, where the subfield of constants is $\mathbb{F}$.

\subsubsection{A principal right ideal ring}

The set of polynomials of the form

$$
\sum_{\text {finie }} \delta^{s} a_{s}
$$

$a_{s} \in F$, is a principal right ideal ring $F[\delta]$. It is commutative if, and only if, $F$ is a field of constants.

\subsection{Input-output system}

A linear system is a finitely generated right $F[\delta]$-module, where $F$ is an inversive difference field ${ }^{5}$. A linear recurrent transducer, or a time-varying convolutional transducer, or a linear input-output system, $\mathcal{T}$ is a system with the following properties:

- there is an input, i.e., a finite subset $\boldsymbol{u}=\left(u_{1}, \ldots, u_{k}\right)$ of $\mathcal{T}$, such that the quotient $\operatorname{module} \mathcal{T} / \operatorname{span}_{F[\delta]}(\boldsymbol{u})$ is torsion. The input will be assumed to be independent, i.e., the module $\operatorname{span}_{F[\delta]}(\boldsymbol{u})$ is free, of rank $k$;

\footnotetext{
${ }^{5}$ This assumption on $F$ being inversive will simplify several further developments. It does not seem to bring any limitation from a practical viewpoint (see, e.g. [26]).
} 
- there is an output, i.e., a finite subset $\boldsymbol{y}=\left(y_{1}, \ldots, y_{n}\right)$ of $\mathcal{T}$;

- $\mathcal{T}$ is causal (cf. [11]), or nonanticipative, i.e., the semi-linear [3] mapping $\delta: \mathcal{T} / \operatorname{span}_{F[\delta]}(\boldsymbol{u}) \rightarrow \mathcal{T} / \operatorname{span}_{F[\delta]}(\boldsymbol{u})$ is injective.

Example 2.2. The transducer $y \delta=u$, i.e., $y(t-1)=u(t)$, where $k=n=1$, should obviously be viewed as non-causal. It is also non-causal in our abstract setting. As a matter of fact the quotient $\operatorname{module} \mathcal{T} / \operatorname{span}_{F[\delta]}(u)$ is a 1-dimensional $F$-vector space spanned by an element corresponding to $u(t+1)$, which is mapped to 0 by $\delta$.

When $F$ is a field of constants, a linear recurrent transducer is called a fixed, or time-invariant, convolutional transducer.

\subsection{State-variable representation}

When viewed as a $F$-vector space, the finitely generated torsion module $\mathcal{T} / \operatorname{span}_{F[\delta]}(\boldsymbol{u})$ is of finite dimension, $m$. Take a basis $\boldsymbol{\xi}=\left(\xi_{1}, \ldots, \xi_{m}\right)$. The next lemma is clear.

Lemma 2.3. $\boldsymbol{\xi} \delta$ is also a basis.

Corollary 2.4. $\boldsymbol{\xi}=\boldsymbol{\xi} \delta A, A \in F^{m \times m}, \operatorname{det}(A) \neq 0$.

Take in $\mathcal{T}$ a $m$-tuple $\boldsymbol{\eta}=\left(\eta_{1}, \ldots, \eta_{m}\right)$ the image of which in $\mathcal{T} / \operatorname{span}_{F[\delta]}(\boldsymbol{u})$ is $\boldsymbol{\xi}$. Then Corollary 2.4 yields a generalised state-variable representation of the transducer $\mathcal{T}$

$$
\begin{aligned}
& \boldsymbol{\eta}=\boldsymbol{\eta} \delta A+\sum_{\mu=0}^{\nu} \boldsymbol{u} \delta^{\mu} \bar{B}_{\mu} \\
& \boldsymbol{y}=\boldsymbol{\xi} \bar{C}+\sum_{\text {finite }} \boldsymbol{u} \delta^{\iota} \bar{D}_{\iota}
\end{aligned}
$$

$\bar{B}_{\mu} \in F^{k \times m}, \bar{C} \in F^{m \times n}, \bar{D}_{\iota} \in F^{k \times n}$. Let $\boldsymbol{\xi}^{\prime}$ be another basis of $\mathcal{T} / \operatorname{span}_{F[\delta]}(\boldsymbol{u})$. Thus $\boldsymbol{\xi}^{\prime}=\boldsymbol{\xi} P, P \in F^{m \times m}$, $\operatorname{det}(P) \neq 0$. Take a $m$-tuple $\boldsymbol{\eta}^{\prime}=\left(\eta_{1}^{\prime}, \ldots, \eta_{m}^{\prime}\right)$ in $\mathcal{T}$ the image of which in $\mathcal{T} / \operatorname{span}_{F[\delta]}(\boldsymbol{u})$ is $\boldsymbol{\xi}^{\prime}$. Then

$$
\boldsymbol{\eta}^{\prime}=\boldsymbol{\eta}+\sum_{\text {finite }} \boldsymbol{u} \delta^{\iota} Q_{\iota}
$$

$Q \in F^{k \times m}$. Note that (2.4) is input-dependent. If, in (2.2), $\nu \geq 2$ and $\bar{B}_{\nu} \neq 0$, set

$$
\boldsymbol{\eta}=\tilde{\boldsymbol{\eta}}-\boldsymbol{u} \delta^{\nu-1}\left(\bar{B}_{\nu} A^{-1} \delta^{-1}\right)
$$

It yields

$$
\tilde{\boldsymbol{\eta}}=\tilde{\boldsymbol{\eta}} \delta A+\sum_{\mu=0}^{\nu-1} \boldsymbol{u} \delta^{\mu} \tilde{B}_{\mu}
$$

If $\bar{B}_{0} \neq 0$, setting

$$
\tilde{\boldsymbol{\eta}}=\overline{\boldsymbol{\eta}}+\boldsymbol{u} \bar{B}_{0}
$$

yields

$$
\overline{\boldsymbol{\eta}}=\overline{\boldsymbol{\eta}} \delta+\sum_{\mu=1}^{\nu-1} \boldsymbol{u} \delta^{\mu} \bar{B}_{\mu}
$$

We have proved the following theorem which is a time-varying generalisation of [11]. 
Theorem 2.5. A causal linear recurrent transducer may be given the Kalman state-variable representation

$$
\begin{aligned}
& \boldsymbol{x}=\boldsymbol{x} \delta A+\boldsymbol{u} \delta B \\
& \boldsymbol{y}=\boldsymbol{x} C+\sum_{\text {finite }} \boldsymbol{u} \delta^{\iota} D_{\iota}
\end{aligned}
$$

where $\boldsymbol{x}=\left(x_{1}, \ldots, x_{m}\right), m=\operatorname{dim}_{F}\left(\mathcal{T} / \operatorname{span}_{F[\delta]}(\boldsymbol{u})\right), A \in F^{m \times m}, \operatorname{det} A \neq 0, B \in F^{k \times m}, C \in F^{m \times n}, D_{\iota} \in$ $F^{k \times m}$.

Remark 2.6. Setting $\boldsymbol{x}=\overline{\boldsymbol{x}}-\boldsymbol{u}\left(B A^{-1} \delta^{-1}\right)$ yields $\overline{\boldsymbol{x}}=\overline{\boldsymbol{x}} \delta A+\boldsymbol{u}\left(B A^{-1} \delta^{-1}\right)$ which might also be interesting in some applications.

\subsection{Controllability and observability}

\subsubsection{Controllability}

The transducer $\mathcal{T}$ is called controllable if, and only if, the module $\mathcal{T}$ free. The next result is an extension to (2.5) of the classic Kalman controllability criterion (compare with [43]):

Proposition 2.7. The transducer $\mathcal{T}$ is controllable if, and only if, the matrix

$$
\left(B, B \delta A, \ldots, B(\delta A)^{m-1}\right)
$$

is of rank $m$.

Proof. It is easy to check that $\operatorname{rk}\left(B, B \delta A, \ldots, B(\delta A)^{m-1}\right)<m$ is equivalent to the existence of a nontrivial torsion submodule of $\mathcal{T}$.

\subsubsection{Observability}

The transducer $\mathcal{T}$ is called observable if, and only if, the modules $\mathcal{T}$ and $\operatorname{span}_{F[\delta]}(\boldsymbol{u}, \boldsymbol{y})$ coincide. The next result is an extension to $(2.5,2.6)$ of the classic Kalman observability criterion (compare with [43]):

Proposition 2.8. The transducer $\mathcal{T}$ is observable if, and only if, the matrix

$$
\left({ }^{t} C,{ }^{t} C \delta^{t} A^{-1}, \ldots,{ }^{t} C\left(\delta^{t} A^{-1}\right)^{m-1}\right)
$$

where ${ }^{t} \bullet$ indicates the transpose matrix, is of rank $m$.

Proof. Utilize $\boldsymbol{x} \delta=\boldsymbol{x} A^{-1}-\boldsymbol{u} \delta B A^{-1}$ for expressing $\boldsymbol{y} \delta^{\iota}, \iota=1, \ldots, m-1$, as $F$-linear combinations of the components of $\boldsymbol{x}$ and $\boldsymbol{u} \delta^{\kappa}, \kappa \geq 0$.

Remark 2.9. By utilizing the inverse $A \delta^{-1}$ of $\delta A^{-1}$, Proposition 2.8 becomes

$$
\operatorname{rk}\left({ }^{t} C,{ }^{t} C^{t} A \delta^{-1}, \ldots,{ }^{t} C\left({ }^{t} A \delta^{-1}\right){ }^{m-1}\right)=m .
$$

\subsection{Transfer matrices}

\subsubsection{Definition}

Let $F(\delta)$ be the quotient field of $F[\delta]$ which is a right Ore ring. The right $F(\delta)$-vector space $\hat{\mathcal{T}}=\mathcal{T} \otimes_{F[\delta]} F(\delta)$ is called the transfer vector space of $\mathcal{T}[12]$. The $F[\delta]$-linear mapping $\mathcal{T} \rightarrow \hat{\mathcal{T}}, \tau \mapsto \hat{\tau}=\tau \otimes 1$, is the (formal) Laplace transform [12]. Its kernel is the torsion submodule of $T$. It is thus injective if, and only if, the module $\mathcal{T}$ is free. As $\boldsymbol{u}$ is independent, $\hat{\boldsymbol{u}}=\left(\hat{u}_{1}, \ldots, \hat{u}_{k}\right)$ is a basis of $\hat{\mathcal{T}}$. It yields

$$
\hat{\boldsymbol{y}}=\left(\hat{y}_{1}, \ldots, \hat{y}_{n}\right)=\hat{\boldsymbol{u}} G
$$


where $G \in F(\delta)^{m \times n}$ is the rational transfer matrix, or the rational generating matrix, of the transducer (compare with [28]). When $k=n=1, G$ is called a rational transfer, or generating function.

Remark 2.10. Note that the dimension of $\hat{\mathcal{T}}$ is equal to the rank of $\mathcal{T}$.

Any element of $F(\delta)$ may be written as a Laurent series $\sum_{\nu>\nu_{0}} \delta^{\nu} a_{\nu}, a_{\nu} \in F, \nu_{0} \in \mathbb{Z}$. It is said to be causal if, and only if, $\nu_{0} \geq 0$. The matrix $G$ is said to be causal if, and only if, all its entries are causal.

Theorem 2.11. Any causal linear recurrent transducer possesses a rational causal transfer matrix. Conversely, any rational causal matrix is the transfer matrix of a causal linear recurrent transducer, which is controllable and observable.

Proof. The first part is an immediate consequence of the definition of causality in Section 2.2 and of the inputoutput relation (2.7). For the second part, utilize the right coprime factorization $G=N D^{-1}, N \in F[\delta]^{k \times n}$, $D \in F[\delta]^{n \times n}$, where $D$ is invertible (see [12]). The transfer matrix of the transducer $\boldsymbol{y} D=\boldsymbol{u} N$, which is both controllable and observable (see [12]), is $G$.

\subsubsection{Interconnection}

Let $h_{v}: \Sigma \rightarrow \mathcal{S}_{v}, v \in \Upsilon$, be a morphism of systems, i.e., of finitely generated right $F[\delta]$-modules. The corresponding fibered sum is a system interconnection (cf. [14]). The parallel interconnection

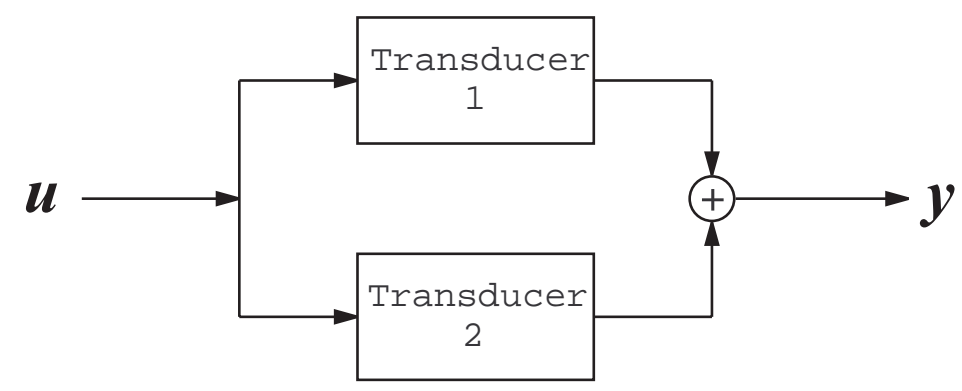

and the series interconnection

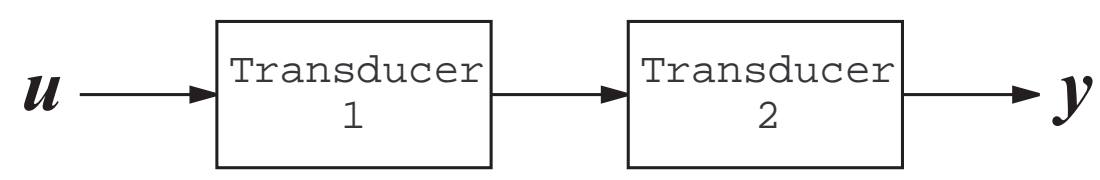

are particular instances of system interconnections. The proof of the following result is straightforward.

Proposition 2.12. The transfer matrix of the parallel (resp. series) interconnection of linear recurrent transducers is the sum (resp. product) of the transfer matrices.

Remark 2.13. Interconnections as simple as those in Proposition 2.12 may lead to a lost of controllability or observability $^{6}$ which is not readable via transfer matrices [14].

\subsection{Input-output inversion}

\subsubsection{General results}

The output rank [8] of the transducer $\mathcal{T}$ is $\varrho=\operatorname{rk}\left(\operatorname{span}_{F[\delta]}(\boldsymbol{y})\right)$. Obviously $0 \leq \varrho \leq \min (k, n)$. The transducer $\mathcal{T}$ is said to be right invertible (resp. left invertible) if, and only if, $\varrho=k$ (resp. $\varrho=n$ ).

\footnotetext{
${ }^{6}$ The continuous-time examples and the results in [14] (see also the references therein) may trivially be adapted to our discretetime context.
} 
Proposition 2.14. $\mathcal{T}$ is right invertible, if and only if, the quotient module $\mathcal{T} / \operatorname{span}_{F[\delta]}(\boldsymbol{y})$ is torsion.

Proof. $\operatorname{rk}\left(\mathcal{T} / \operatorname{span}_{F[\delta]}(\boldsymbol{y})\right)=\operatorname{rk}(\mathcal{T})-\varrho$. Since $\mathcal{T} / \operatorname{span}_{F[\delta]}(\boldsymbol{u})$ is torsion, $\operatorname{rk}(\mathcal{T})=\operatorname{rk}\left(\operatorname{span}_{F[\delta]}(\boldsymbol{u})=k\right.$. Thus $\operatorname{rk}\left(\mathcal{T} / \operatorname{span}_{F[\delta]}(\boldsymbol{y})\right)=0$ if, and only if, $\varrho=k$.

In a more down to earth language, Lemma 2.14 means that $\boldsymbol{u}$ may be obtained from $\boldsymbol{y}$ thanks to difference equations. The example $y=u \delta$, where $k=n=1$, shows that the right inverse transducer is not generally causal. Left invertibility means that the components of $\boldsymbol{y}$ are $F[\delta]$-linearly independent.

The next proposition is an immediate consequence of Remark 2.10 .

Proposition 2.15. The linear recurrent transducer $\mathcal{T}$ is right (resp. left) invertible if, and only if, its transfer matrix is right (resp. left) invertible.

Corollary 2.16. If the linear recurrent transducer $\mathcal{T}$ is right (resp. left) invertible, then $n \geq k$ (resp. $n \leq k$ ).

If $k=n$, the transducer is said to be square. Then right and left invertibilities coincide. An invertible square transducer is right and left invertible.

\subsubsection{Encoders}

A linear recurrent transducer, which is right invertible, is called a linear recurrent encoder, or a (time-varying) convolutional encoder. If $F$ is a field of constants, it is called a (fixed) convolutional encoder ${ }^{7}$. A square encoder is called a linear recurrent encrypter.

\subsection{Some useful constructions}

\subsubsection{Blocking}

For any integer $\Omega>1, F\left[\delta^{\Omega}\right] \subset F[\delta]$. Thus any right $F[\delta]$-module $\boldsymbol{M}$ may also be viewed as a right $F\left[\delta^{\Omega}\right]$-module $\boldsymbol{M}_{\Omega}$ called the $\Omega^{\text {th }}$-blocking, or $\Omega^{\text {th }}$-interleaving, module.

Lemma 2.17. $\operatorname{rk}\left(\boldsymbol{M}_{\Omega}\right)=\Omega \operatorname{rk}(\boldsymbol{M})$.

Proof. If $\xi_{1}, \ldots, \xi_{\ell}$ are $F[\delta]$-linearly independent elements in $\boldsymbol{M}$, then $\xi_{1}, \xi_{1} \delta, \ldots, \xi_{1} \delta^{\Omega-1}, \ldots, \xi_{\ell}, \xi_{\ell} \delta, \ldots, \xi_{\ell} \delta^{\Omega-1}$ are $F\left[\delta^{\Omega}\right]$-linearly independent.

The $\Omega^{\text {th }}$-blocking transducer, or $\Omega^{\text {th }}$-interleaving transducer, $\mathcal{T}_{\Omega}$ of $\mathcal{T}$ is the linear recurrent transducer defined by

- its module is the $\Omega^{\text {th }}$-blocking module $\mathcal{T}_{\Omega}$;

- its input and output are respectively $\left(\boldsymbol{u}, \boldsymbol{u} \delta, \ldots, \boldsymbol{u} \delta^{\Omega-1}\right)$ and $\left(\boldsymbol{y}, \boldsymbol{y} \delta, \ldots, \boldsymbol{y} \delta^{\Omega-1}\right)$.

The next result is clear:

Proposition 2.18. If $\mathcal{T}$ is controllable (resp. observable, right invertible, left invertible), then $\mathcal{T}_{\Omega}$ is also controllable (resp. observable, right invertible, left invertible).

\subsubsection{Puncturing}

Puncturing a linear recurrent transducer $\mathcal{T}$ means taking a linear recurrent transducer $\mathcal{T}_{P}$ defined by the same module, the same input and by an output which is a subset of $\boldsymbol{y}$. The next result is clear:

Proposition 2.19. If $\mathcal{T}$ is controllable (resp. left invertible), then $\mathcal{T}_{P}$ is also controllable (resp. left invertible). If $\mathcal{T}$ is observable (resp. right invertible), then $\mathcal{T}_{P}$ is not necessarily observable (resp. right invertible).

\footnotetext{
${ }^{7}$ Even if $F$ is a finite field, there exists several definitions of convolutional encoders in the existing literature.
} 


\section{Some Properties of LINEAR RECURRENT CODES}

\subsection{Equivalence of encoders and codes}

\subsubsection{Equivalence}

Two linear recurrent encoders with inputs $\boldsymbol{u}=\left(u_{1}, \ldots, u_{k}\right), \boldsymbol{u}^{\prime}=\left(u_{1}, \ldots, u_{k^{\prime}}^{\prime}\right)$ and outputs $\boldsymbol{y}=\left(y_{1}, \ldots, y_{n}\right)$, $\boldsymbol{y}^{\prime}=\left(y_{1}^{\prime}, \ldots, y_{n^{\prime}}^{\prime}\right)$ are said to be equivalent if, and only if, the following conditions are satisfied:

(1) $n=n^{\prime}$;

(2) there exists $\sigma \in \mathrm{S}_{n}$, where $\mathrm{S}_{n}$ is the symmetric group over $\{1, \ldots, n\}$, such that the mapping $y_{\iota} \mapsto y_{\iota \sigma}^{\prime}$, $\iota=1, \ldots, n$, defines an isomorphism between the modules $\operatorname{span}_{F[\delta]}(\boldsymbol{y})$ and $\operatorname{span}_{F[\delta]}\left(\boldsymbol{y}^{\prime}\right)$.

Proposition 3.1. The inputs of two equivalent linear recurrent encoders possess the same number of components.

Proof. Let $\varrho$ and $\varrho^{\prime}$ be the output ranks of the encoders $\mathcal{T}$ and $\mathcal{T}^{\prime}$. The right invertibility of $\mathcal{T}$ and $\mathcal{T}^{\prime}$ implies $\varrho=k$ and $\varrho^{\prime}=k^{\prime}$. The equivalence of $\mathcal{T}$ and $\mathcal{T}^{\prime}$ implies $\varrho=\varrho^{\prime}$.

\subsubsection{Codes}

A linear recurrent code, or a (time-varying) convolutional code is an equivalence class between linear recurrent encoders. From Proposition 3.1, we know already two integers $k, n, 0<k \leq n$ which are attached to the code, which is therefore called a $(n, k)$ linear recurrent code. Its rate is $\frac{k}{n}$. By a slight abuse of language, $\operatorname{span}_{F[\delta]}(\boldsymbol{y})$ is sometimes called a linear recurrent code, or a (time-varying) convolutional code. When $F$ is a finite field of constants, a linear recurrent code is called a (fixed) convolutional code. A code is said to be free, or controllable, if, and only if, the module $\operatorname{span}_{F[\delta]}(\boldsymbol{y})$ is free.

\subsection{Syndrome formers}

Let $\mathcal{F}_{n}$ be the free right $F[\delta]$-module, with basis $\bar{y}_{1}, \ldots, \bar{y}_{n}$. The mapping $\bar{y}_{\iota} \mapsto y_{\iota}, \iota=1, \ldots, n$, defines an epimorphism $\mathcal{F}_{n} \rightarrow \operatorname{span}_{F[\delta]}(\boldsymbol{y})$ and the short exact sequence

$$
0 \rightarrow \mathcal{F}_{n-k} \rightarrow \mathcal{F}_{n} \rightarrow \operatorname{span}_{F[\delta]}(\boldsymbol{y}) \rightarrow 0
$$

where $\mathcal{F}_{n-k}$ a free right $F[\delta]$-module of rank $n-k$. A syndrome former of the code is a presentation matrix of $\operatorname{span}_{F[\delta]}(\boldsymbol{y})$, which corresponds here to the monomorphism $\mathcal{F}_{n-k} \rightarrow \mathcal{F}_{n}$.

The sequence (3.1) splits, i.e., $\mathcal{F}_{n} \simeq \mathcal{F}_{n-k} \oplus \operatorname{span}_{F[\delta]}(\boldsymbol{y})$, if, and only if, the code is free.

\subsection{Some properties of free codes}

From now on and until the end of the paper codes are assumed to be free ${ }^{8}$. When $F$ is a finite field of constants, a (fixed) convolutional code may be defined as a certain $F[\delta]$-submodule of the $F[\delta]$-module $\mathcal{L}=\left\{\sum_{v \geq 0} \delta^{v} a_{1 v}, \ldots, \sum_{v \geq 0} \delta^{v} a_{n v}\right\}$ of $n$-tuple of formal power series. The relationship with our approach ${ }^{9}$ is given the $F[\delta]$-module Hom $\left(\operatorname{span}_{F[\delta]}(\boldsymbol{y}), \mathcal{L}\right)$ of $F[\delta]$-module morphisms $\Phi=\left(\phi_{1}, \ldots, \phi_{n}\right): \operatorname{span}_{F[\delta]}(\boldsymbol{y}) \rightarrow \mathcal{L}$, $\left(y_{1}, \ldots, y_{n}\right) \mapsto\left(y_{1} \phi, \ldots, y_{n} \phi\right)$ (compare with [38]).

\subsubsection{Dual codes and parity check matrices}

The image of $\mathcal{F}_{n-k}$ in $\mathcal{F}_{n}$ is called the dual code. A syndrome former of the dual code is called a parity check matrix of the code.

Remark 3.2. When $F$ is a finite field of constants, the dual code of a convolutional code is usually defined as for block codes via an orthogonality relation. We leave to the reader to construct explicitly the relationship with our definition.

\footnotetext{
${ }^{8}$ When $F$ is a finite field of constants, a (fixed) convolutional code is often defined as a vector subspace of $F(\delta){ }^{1 \times n}($ see, e.g. $[26,34])$. With respect to this transfer matrix setting the freeness may always be assumed.

${ }^{9}$ This is more generally the relationship (see [10]) between our module-theoretic setting and Willems' behavioral approach [44].
} 


\subsubsection{Polynomial and basic encoders}

A controllable and observable encoder $\mathcal{E}$ is said to be polynomial if, and only if, $\boldsymbol{u}$ is a basis of the free module $\mathcal{E}$. The next property is an immediate consequence of Theorem 2.11:

Proposition 3.3. A controllable and observable encoder is polynomial if, and only if, the entries of its transfer matrix are polynomial, i.e., belong to $F[\delta]$.

The polynomial encoder $\mathcal{E}$ is said to be basic if, and only if, $\mathcal{E}=\operatorname{span}_{F[\delta]}(\boldsymbol{y})$. By taking for $\boldsymbol{u}$ any basis of the free $\operatorname{module} \operatorname{span}_{F[\delta]}(\boldsymbol{y})$ we obtain the

Proposition 3.4. Any free code admits a basic encoder.

\subsubsection{Systematic encoders}

Proposition 3.5. Any free code admits a systematic encoder, i.e., an encoder where $k$ components of the output are identical to the $k$ components of the input.

Proof. The result is clear if $k=n ; \boldsymbol{y}$ is a basis of $\operatorname{span}_{F[\delta]}(\boldsymbol{y})$ and can be taken as an input. Assume that the result holds for $n=n_{0} \geq k$. Take $n=n_{0}+1$. Since the components of $\boldsymbol{y}$ are $F[\delta]$-linearly dependent we may write

$$
y_{1} \gamma_{1}+\cdots+y_{n_{0}+1} \gamma_{n_{0}+1}=0
$$

where $\gamma_{1}, \ldots, \gamma_{n_{0}+1} \in F[\delta]$ are right coprime. At least one of the coefficients $\gamma_{\iota}, \iota=1, \ldots, n_{0}+1, \gamma_{n_{0}+1}$ for instance, when expressed as a sum (2.1), is such that $a_{0} \neq 0$. Apply the assumption to the code spanned by $y_{1}, \ldots, y_{n_{0}}$ and utilise the causal relation $y_{n_{0}+1}=-\left(y_{1} \gamma_{1}+\cdots+y_{n_{0}} \gamma_{n_{0}}\right) \gamma_{n_{0}+1}^{-1}$.

\subsubsection{Non-catastrophic encoders}

The ring of Laurent polynomials $F\left[\delta, \delta^{-1}\right]$ is the localized ring of $F[\delta]$ by the multiplicative monoid $\left\{\delta^{s} \mid s\right.$ $\geq 0\}$, which satisfies the right Ore condition. The corresponding localized right $F\left[\delta, \delta^{-1}\right]$-module $\mathcal{E} \otimes_{F[\delta]} F\left[\delta, \delta^{-1}\right]$ of $\operatorname{span}_{F[\delta]}(\boldsymbol{u})$ is free, if $\mathcal{E}$ is controllable. The canonical mapping $\mathcal{E} \rightarrow \mathcal{E} \otimes_{F[\delta]} F\left[\delta, \delta^{-1}\right], v \mapsto v \otimes 1$, being injective, $\mathcal{E}$ may be considered as a subset of $\mathcal{E} \otimes_{F[\delta]} F\left[\delta, \delta^{-1}\right]$. A controllable encoder is said to be non-catastrophic if, and only if, $\boldsymbol{u}$ belongs to $\operatorname{span}_{F[\delta]}(\boldsymbol{y}) \otimes_{F[\delta]} F\left[\delta, \delta^{-1}\right]$. The next result is an immediate consequence of Proposition 3.4.

Proposition 3.6. Any free code admits a non-catastrophic encoder.

\subsection{Forney's theorem}

\subsubsection{An important filtration}

Define a filtration of $F[\delta]$ by setting $\mathbf{F}_{\alpha}=\left\{P \delta^{\alpha}\right\}, \alpha \geq 0, P \in F[\delta]$. Thus $F[\delta]=\mathbf{F}_{0} \supset \mathbf{F}_{1} \supset \ldots$ The corresponding filtration for the free module $\operatorname{span}_{F[\delta]}(\boldsymbol{y})$ is obtained by setting $\mathbf{C}_{\alpha}=\operatorname{span}_{F[\delta]}(\boldsymbol{y}) \mathbf{F}_{\alpha}$. Thus $\operatorname{span}_{F[\delta]}(\boldsymbol{y})=\mathbf{C}_{0} \supset \mathbf{C}_{1} \supset \ldots$ Any element $x \in \operatorname{span}_{F[\delta]}(\boldsymbol{y})$ may be written uniquely as a finite sum

$$
x=\sum_{\alpha=\nu}^{\mu} \xi_{\alpha} \delta^{\alpha}
$$

where $\xi_{\alpha} \delta^{\alpha}$ is homogeneous, of weight $\alpha$ ( $\xi_{0}$ is homogeneous of weight 0$)$. The element $x$ is said to be of order $\nu$ (resp. degree $\mu$ ) if, and only if, $\xi_{\nu} \neq 0$ (resp. $\xi_{\mu} \neq 0$ ). It is homogeneous if, and only if, $\nu=\mu$. The next results are clear.

Lemma 3.7. The semi-linear linear mapping $\delta^{\ell}: \boldsymbol{C}_{\alpha} \rightarrow \boldsymbol{C}_{\alpha+\ell}, \ell>0$, is bijective.

Corollary 3.8. For any homogeneous element $x_{\alpha+\ell}$ of order $\alpha+\ell$ there exists a homogeneous element $x_{\alpha}$ of order $\alpha$ such that $x_{\alpha} \delta^{\ell}=x_{\alpha+\ell}$. 
Lemma 3.9. Homogeneous elements of order $\nu$ are $F[\delta]$-linearly independent if, and only if, they are $F$-linearly independent.

Corollary 3.10. The F-vector space $\boldsymbol{C}_{\alpha} / \boldsymbol{C}_{\alpha+1}$ is of dimension $k$.

\subsubsection{The result}

Let $\varepsilon_{1}$ be the highest degree of the components of $\boldsymbol{y}$, when written as in (3.3). Let $V_{1}$ be the $\varpi_{1}$-dimensional $F$-vector space spanned by the corresponding homogeneous elements. Choose according to Corollary 3.8 homogeneous elements $u_{1}, \ldots, u_{\varpi_{1}}$, of degree 0 , such that $V_{1}=\operatorname{span}\left(u_{1} \delta^{\varepsilon_{1}}, \ldots, u_{\varpi_{1}} \delta^{\varepsilon_{1}}\right)$. Let $\varepsilon_{2}<\varepsilon_{1}$ be the first integer such that $u_{1} \delta^{\varepsilon_{2}}, \ldots, u_{\varpi_{1}} \delta^{\varepsilon_{2}}$ does not span the $F$-vector space spanned by the homogeneous components of order $\varepsilon_{2}$ in $\boldsymbol{y}$. Complete then $u_{1}, \ldots, u_{\varpi_{1}}$ as above. We obtain a basis $\boldsymbol{u}=\left(u_{1}, \ldots, u_{m}\right)$ and a corresponding polynomial transfer matrix with lines of degrees ${ }^{10} e_{1} \leq e_{2} \leq \cdots \leq e_{k}$.

We must show that the above basic encoder is minimal, i.e., that the degrees $f_{1} \leq f_{2} \leq \cdots \leq f_{k}$ of the lines of any polynomial generating matrix verify $e_{\iota} \leq f_{\iota}, \iota=1, \ldots, k$. The next lemma, which is obvious, demonstrates that this result holds true if $k=1$.

Lemma 3.11. Take a free $F[\delta]$-module $M$ of rank 1 . Two bases $b$ and $b^{\prime}$ are related by $b=b^{\prime} \gamma, \gamma \in F, \gamma \neq 0$. Let $N \supseteq M$ be another free $F[\delta]$-module of rank 1 . Then, for any basis $c$ of $N, b=b \pi, \pi \in F[\delta]$.

By considering the quotient module $\operatorname{span}_{F[\delta]}(\boldsymbol{y}) / \operatorname{span}_{F[\delta]}\left(u_{1}\right)$, which is free of rank $k-1$, we obtain the minimality for any $k \geq 2$, assuming that it holds true for $k-1$.

We have proved:

Theorem 3.12. For any free linear recurrent code, there exists a basic encoder, called minimal, such that the degrees of the lines of its transfer matrix are $e_{1} \leq e_{2} \leq \cdots \leq e_{k}$. The degrees $f_{1} \leq f_{2} \leq \cdots \leq f_{k}$ of the lines of a transfer matrix of any equivalent polynomial encoder verify $e_{\kappa} \leq f_{\kappa}, \kappa=1, \ldots, k$.

A corresponding input is called a Forney input.

\section{A connection Between Convolutional And Block Codes}

From now on $F$ is a finite field $\mathbb{F}_{q}$ of constants. We will therefore be working with free (fixed) linear convolutional codes.

\subsection{Sliding block codes}

A sliding presentation of a free $(n, k)$ linear convolutional code is given by a submodule $\mathrm{C}$ of rank $k$ of a free $\mathbb{F}_{q}[\delta]$-module $\mathrm{E}$ of rank $n$ such that the quotient module $\mathrm{E} / \mathrm{C}$ is free ${ }^{11}$. The sliding (linear) block code of order $\Omega$ of a given sliding presentation is given by the $\mathbb{F}_{q}$-vector subspace $\mathrm{C} / \mathrm{C} \delta^{\Omega}$ of the $\mathbb{F}_{q}$-vector space $\mathrm{E} / \mathrm{E} \delta^{\Omega}$. It is obviously a $(n \Omega, k \Omega)$ block code.

Theorem 4.1. For integers $n, k, \Omega, 1 \leq k<n, \Omega \geq 1$, there exists a free $(n, k)$ convolutional code with a sliding presentation such that its sliding block code of order $\Omega$ is an arbitrary $(n \Omega, k \Omega)$ block code.

Proof. Take an arbitrary $(n \Omega, k \Omega)$ block code defined by a $k \Omega$-dimensional subspace $U$ of a $n \Omega$-dimensional of a $\mathbb{F}_{q}$-vector space $Y$. For any integer $\nu \geq 0$, set $Y \delta^{\nu \Omega}=\left\{y \delta^{\nu \Omega} \mid y \in E\right\}$. Define the free $\mathbb{F}_{q}\left[\delta^{\Omega}\right]$-modules $\mathrm{U}=\bigoplus_{\nu \geq 0} U \delta^{\nu \Omega} \subset \mathrm{Y}=\bigoplus_{\nu \geq 0} Y \delta^{\nu \Omega}$. Consider now the free $\mathbb{F}_{q}[\delta]$-modules $\mathbb{F}_{q}[\delta] \bigotimes_{\mathbb{F}_{q}\left[\delta^{\Omega}\right]} \mathrm{U} \subset \mathbb{F}_{q}[\delta] \bigotimes_{\mathbb{F}_{q}\left[\delta^{\Omega}\right]} \mathrm{Y}$.

Any basis $\underline{\boldsymbol{u}}=\left(\underline{u}_{1}, \ldots, \underline{u}_{k \Omega}\right)$ of $U$ may be viewed as a basis of $\mathrm{U}$. By considering a systematic presentation of the block code, we may complete some basis $\underline{\boldsymbol{u}}$ as a basis $\underline{\boldsymbol{y}}=\left(\underline{y}_{1}, \ldots, \underline{y}_{n \Omega}\right)$ of $Y$; $\underline{\boldsymbol{y}}$ may also be viewed as a basis of Y. Take a partition of $\underline{\boldsymbol{u}}$ consisting of $k$ disjoint sets of $\Omega$ elements. Complete it as a partition of $\underline{\boldsymbol{y}}$ of $n$ disjoint sets of $\Omega$ elements. For the subsets $\left\{\left(z_{1}^{\iota}, \ldots, z_{\Omega}^{\iota}\right) \mid \iota=1, \ldots, n\right.$ of the partition, define the submodule $\mathrm{P}=\operatorname{span}_{\mathbb{F}_{q}[\delta]}\left(\left\{z_{\kappa}^{\iota} \delta-z_{\kappa+1}^{\iota} \mid \iota=1, \ldots, n ; \kappa=1, \ldots, \Omega-1\right\}\right)$ of $\mathbb{F}_{q}[\delta] \bigotimes_{\mathbb{F}_{q}\left[\delta^{\Omega}\right]} \mathrm{U} \subset \mathbb{F}_{q}[\delta] \bigotimes_{\mathbb{F}_{q}\left[\delta^{\Omega}\right]} \mathrm{Y}$.

\footnotetext{
${ }^{10}$ The degree of a line is the maximum degree of its entries.

${ }^{11} \mathrm{This}$ is an immediate consequence of the splitting property of the short exact sequence (3.1).
} 
Lemma 4.2. The quotient $\mathbb{F}_{q}[\delta]$-module $E=\mathbb{F}_{q}[\delta] \bigotimes_{\mathbb{F}_{q}\left[\delta^{\delta}\right]} Y / P$ is free of rank $n$. The canonical image $C$ of $\mathbb{F}_{q}[\delta] \bigotimes_{\mathbb{F}_{q}\left[\delta^{\Omega}\right]} U$ into $\mathbb{F}_{q}[\delta] \bigotimes_{\mathbb{F}_{q}\left[\delta^{\Omega}\right]} Y / P$ is free of rank $k$.

Proof. A basis of $\mathrm{E}$ is $\left\{z_{1}^{\iota} \mid \iota=1, \ldots, n\right\}$.

The solution is given by the sliding presentation $\mathrm{C} \subset \mathrm{E}$.

\subsection{Sketch of a feedback decoding procedure}

The $\mathbb{F}_{q}\left[\delta, \delta^{-1}\right]$-modules $\mathbb{F}_{q}\left[\delta, \delta^{-1}\right] \otimes_{\mathbb{F}_{q}[\delta]} \mathrm{C}$ and $\mathbb{F}_{q}\left[\delta, \delta^{-1}\right] \otimes_{\mathbb{F}_{q}[\delta]}$ E may be seen as $\mathbb{F}_{q}[\delta]$-modules $\overline{\mathrm{C}}$ and $\overline{\mathrm{E}}$. For any integer $\alpha \geq 0$, the $(\alpha+1)^{\text {th }}$ frame ${ }^{12}$ is the block code is given by the $\mathbb{F}_{q^{-}}$vector subspace $\overline{\mathrm{C}} \delta^{(-\alpha-\Omega)} / \overline{\mathrm{C}} \delta^{-\alpha}$ of the $\mathbb{F}_{q^{-}}$vector space $\overline{\mathrm{E}} \delta^{(-\alpha-\Omega)} / \overline{\mathrm{E}} \delta^{-\alpha}$. It is clear that all those frames are isomorphic to the sliding block code of order $\Omega$.

A decoding procedure of any frame will of course take advantage of the nature of the sliding block code. Comparing the results for the $\beta^{\text {th }}$ and $(\beta+\ell)^{\text {th }}, \beta, \ell \geq 1$, permits some checking if $\ell<\Omega$. This feedback type decoding of the convolutional code may be enriched by some concatenations (see, e.g. $[2,4,30]$ ) of the frames.

\section{REFERENCES}

[1] C. Berrou and A. Glavieux, Near-optimum error-correcting coding and decoding: Turbo-codes. IEEE Trans. Communicat. 44 (1996) 1261-1271.

[2] R.E. Blahut, Theory and Practice of Error Control Codes. Addison-Wesley (1983).

[3] N. Bourbaki, Algèbre, Chap. 2. Hermann (1970).

[4] G. Cohen, J.-L. Dornstetter and P. Godlewski, Codes correcteurs d'erreurs. Masson (1992).

[5] R.M. Cohn, Difference Algebra. Interscience (1965).

[6] A. Dholakia, Introduction to Convolutional Codes with Applications. Kluwer (1994).

[7] F. Fagnani and S. Zampieri, System-theoretic properties of convolutional codes over rings. IEEE Trans. Inform. Theory $\mathbf{4 7}$ (2001) 2256-2274.

[8] M. Fliess, Automatique en temps discret et algèbre aux différences. Forum Math. 2 (1990) 213-232.

[9] M. Fliess, Some basic structural properties of generalized linear systems. Systems Control Lett. 15 (1990) 391-396.

[10] M. Fliess, A remark on Willems' trajectory characterization of linear controllability. Systems Control Lett. 19 (1992) $43-45$.

[11] M. Fliess, Reversible linear and nonlinear discrete-time dynamics. IEEE Trans. Automat. Control 37 (1992) 1144-1153.

[12] M. Fliess, Une interprétation algébrique de la transformation de Laplace et des matrices de transfert. Linear Algebra Appl. 203-204 (1994) 429-442.

[13] M. Fliess, Variations sur la notion de contrôlabilité, in Journée Soc. Math. France. Paris (2000) 47-86.

[14] M. Fliess and H. Bourlès, Discussing some examples of linear system interconnections. Systems Control Lett. 27 (1996) 1-7.

[15] M. Fliess, J. Lévine, P. Martin and P. Rouchon, Flatness and defect of non-linear systems: Introductory theory and applications. Internat. J. Control 61 (1995) 1327-1361.

[16] M. Fliess and R. Marquez, Continuous-time linear predictive control and flatness: A module-theoretic setting with examples. Internat. J. Control 73 (2000) 606-623.

[17] M. Fliess and R. Marquez, Une approche intrinsèque de la commande prédictive linéaire discrète. APII J. Europ. Syst. Automat. 35 (2001) 127-147.

[18] M. Fliess, R. Marquez, E. Delaleau and H. Sira-Ramírez, Correcteurs proportionnels-intégraux généralisés. ESAIM: COCV 7 (2002) 23-41.

[19] M. Fliess, R. Marquez and H. Mounier, An extension of predictive control, PID regulators and Smith predictors to some linear delay systems. Internat. J. Control (to appear).

[20] M. Fliess and H. Mounier, Controllability and observability of linear delay systems: An algebraic approach. ESAIM: COCV 3 (1998) 301-314.

[21] G.D. Forney Jr., Convolutional codes I: Algebraic structure. IEEE Trans. Inform. Theory 16 (1970) 720-738.

[22] G.D. Forney Jr., Minimal bases of rational vector spaces, with applications to multivariable linear systems. SIAM J. Control 13 (1975) 493-520.

[23] G.D. Forney Jr., Algebraic structure of convolutional codes and algebraic system theory, in Mathematical System Theory The Influence of R.E. Kalman, edited by A.C. Antoulas. Springer (1991) 527-557.

\footnotetext{
${ }^{12}$ This mathematical definition of frames, where the previous codewords are subtracted, differs from the usual meaning in the literature (see, e.g. [2]). Note however that this substraction of previous codewords is asserted in [2] to be of utmost importance in feedback decoding.
} 
[24] G.D. Forney Jr. and M.D. Trott, The dynamics of group codes: State-space, trellis diagrams and canonical encoders. IEEE Trans. Inform. Theory 39 (1993) 1491-1513.

[25] G.D. Forney Jr., B. Marcus, N.T. Sindhushayana and M. Trott, A multilingual dictionary: System theory, coding theory, symbolic dynamics and automata theory, in Different Aspects of Coding Theory. Proc. Symp. Appl. Math. 50; Amer. Math. Soc. (1995) 109-138.

[26] R. Johannesson and K.Sh. Zigangirov, Fundamentals of Convolutional Coding. IEEE Press (1999).

[27] T. Kailath, Linear Systems. Prentice-Hall (1979).

[28] E.W. Kamen, P.P. Khargonekar and K.R. Poola, A transfer-function approach to linear time-varying discrete-time systems. SIAM J. Control Optim. 23 (1985) 550-565.

[29] T.Y. Lam, Lectures on Rings and Modules. Springer (1999).

[30] S. Lin and D.J. Costello Jr., Error Control Coding: Fundamentals and Applications. Prentice-Hall (1983).

[31] J.H. van Lint, Introduction to Coding Theory, $3^{\text {rd }}$ Edition. Springer (1999).

[32] H.-A. Loeliger, G.D. Forney Jr., T. Mittelholzer and M.D. Trott, Minimality and observability of group systems. Linear Algebra Appl. 205-206 (1994) 937-963.

[33] J.L. Massey and M.K. Sain, Codes, automata and contnuous systems: Explicit interconnections. IEEE Trans. Automat. Control 12 (1967) 644-650.

[34] R.J. McEliece, The algebraic theory of convolutional codes, in Handbook of Coding Theory, Vol. 1, edited by V. Pless and W.C. Huffman. Elsevier (1998) 1065-1138.

[35] J.C. McConnel and J.C. Robson, Noncommutative Noetherian Rings. Wiley (1987).

[36] H. Mounier, P. Rouchon and J. Rudolph, Some examples of linear systems with delays. APII J. Europ. Syst. Automat. 31 (1997) 911-925.

[37] P. Piret, Convolutional Codes, an Algebraic Approach. MIT Press (1988).

[38] J. Rosenthal, Connections between linear systems and convolutional codes, in Codes, Systems and Graphical Models, edited by B. Marcus and J. Rosenthal. Springer (2000) 39-66.

[39] J. Rosenthal, J.M. Schumacher and E.V. York, On behaviors and convolutional codes. IEEE Trans. Informat. Theory 42 (1996) 1881-1891.

[40] J. Rosenthal and E.V. York, BCH convolutional codes. IEEE Trans. Inform. Theory 45 (1999) 1833-1844.

[41] J. Rotman, An Introduction to Homological Algebra. Academic Press (1979).

[42] A.J. Viterbi and J.K. Omura, Principles of Digital Communication and Coding. McGraw-Hill (1979).

[43] L. Weiss, Controllability, realization and stability of discrete-time systems. SIAM J. Control 10 (1972) 230-251.

[44] J.C. Willems, Paradigms and puzzles in the theory of dynamical systems. IEEE Trans. Automat. Control 36 (1991) $259-294$.

[45] G. Zémor, Cours de cryptographie. Cassini (2000). 